\title{
Guidelines for Identification of Top-down Cracks (TDC) in In- Service Flexible Pavements
}

\author{
Nirmal Dhakal, Mohammad Bashar, and Mostafa Elseifi* \\ Department of Civil and Environmental Engineering, Louisiana State University, Baton Rouge, LA 70803
}

\begin{abstract}
The objective of this study was to establish guidelines to identify top-down cracking (TDC) in flexible pavements using digital image analysis and the characteristics of these cracks. Past studies indicated that the time after construction of the pavement and location of the cracks are key parameters to identify top-down cracking. The cracks were reported to appear on the wheel path or at the outer edge of the wheel path typically within 3 to 8 years of construction. In-service pavement sections were selected for analysis based on the parameters identified from the literature and computer-vision techniques were employed to investigate the geometric characteristics of these cracks. Based on the results of the analysis, the average crack width was observed to be 3 to $7 \mathrm{~mm}$. With respect to the orientation of the crack, the cracks segments were mostly longitudinal with typical deviation of 20 degrees. The orientation and intensity characteristics of top-down cracks were found as useful features in crack identification.
\end{abstract}

\section{Introduction}

Top- down cracking has been a topic of frequent and continuing discussion among pavement researchers in the past two decades. There is no specific distinction in occurrence of top-down cracking in terms of climatic zones, pavement thicknesses, location in pavement surface, and modes and orientation. In addition, the specific causes of top-down cracking are still debated. Consideration of TDC in pavement design methods is complicated as field characterization of these cracks have not been not well established as compared to fatigue cracking, which initiates at the bottom of the Asphalt Concrete (AC) layer. If TDC assessment can be performed as a part of network-level condition survey, it will facilitate for state agencies to set up a more realistic schedule and budget for repair of these cracks.

The noninvasive evaluation of pavement surface conditions is challenging yet powerful, efficient, and safe for state agencies. Various works have been conducted in recent years based on Ground Penetrating Radar (GPR) and laser technologies to evaluate pavement conditions non-destructively. Though the image processing techniques date back to the 1970s, recent advancements in image acquisition and processing have proven a promising tool to assess flexible pavements in terms of surface deterioration compared to laser or GPR technologies. The image processing language defines crack as a cluster of low intensity pixels compared to the surrounding pavement surface pixels that form an arbitrary line shape in length, width and directions [1]. In addition, other photometric hypothesis considers the gray level distributions of road crack and road surface to be independent. Geometrically, a crack is a thin continuous object consisting of a set of connected segments of different orientation and varying in width along the length. From photometric and geometric point of view, the points inside a crack can be considered as points of interest [2].

There are five different types of image analysis techniques namely histogram analysis, mathematical morphological tools, learning phase, filtering, and analysis of model and these techniques assume different hypothesis. The morphological method adopted in this study is based on the hypothesis that a crack is a thin continuous object with darker pixels compared to pavement surface. The basic five steps considered in morphological method are (i) preprocessing of image, (ii) binarization, (iii) refinement by closing, (iv) segmentation with shape analysis, and (v) feature extraction [2].

In the present study, guidelines were developed to detect top-down cracking non-destructively based on the geometric characteristics of these cracks and image processing techniques.

\section{Objectives}

The primary objective of this study was to develop guidelines for characterization and detection of top down cracks non-destructively in in-service flexible pavements using digital image processing and the geometric characteristics of these cracks.

\section{Background}

\subsection{Top-down cracking}

\footnotetext{
* Corresponding author: elseifi@1su.edu
} 
In past, pavement cracking was assumed to initiate at the bottom of the AC layer and propagate upwards to the surface, i.e., bottom-up crack. In the past two decades, the opposite mode of crack initiation and propagation gained a significant attention amongst researchers and pavement practitioners. Literature suggests that the longitudinal top-down cracks usually appear in the wheel paths due to high surface horizontal tensile stresses due to tire loads while other forms of longitudinal cracks are usually bottom-up. Three main stages of crack propagation have been described by Svasidisant et al. [3]. The first stage starts with the appearance of single short longitudinal cracks just outside the wheel path in the surface of the AC layer. In the second stage, the cracks grow longer, and sister cracks develop within 0.3 to 1.0 meters of the original cracks. Over time, the parallel cracks get interconnected via short transverse cracks, which are described as the third stage of crack development [3].

Myers et al. [4] also reported the location of TDC being just outside the wheel path. They studied pavements with AC thicknesses of 50.8 to $203.2 \mathrm{~mm}$ where the crack depths ranged from $25.4 \mathrm{~mm}$ to the full depth of asphalt layer. The cracks were observed to appear five to ten years after construction. The cracks were mostly longitudinal having crack widths of about 3.05 to $3.81 \mathrm{~mm}$ at the surface and decreasing with depth [4]. The time required for the cracks to appear has been reported by Svasidisant et al. [3] to be varying from one to five years. The study showed that TDC had propagated through the entire AC layer in a 15-year-old pavement with rubblized base.

Uhlmeyer et al. [5] reported that for structurally sound pavements that were designed for acceptable equivalent single axle loads, the TDC typically occurred after 3 to 8 years of construction. The service life before appearance of TDC were observed be 1 to 5 years for Japan, 3 to 5 years for France, 5 to 10 years for Florida, and up to 10 years for the UK. In a study for Washington DOT, they observed that the thick AC were also susceptible to TDC and concluded that these cracks would randomly stretch when the thickness of the asphalt layers exceeded 160 to $180 \mathrm{~mm}$ [5].

\subsection{Image processing}

Maser [6] proposed a threshold-based segmentation for image analysis by enhancing the image using histogram equalization [6]. Georgopoulos et al. [7] developed an algorithm to automatically identify the type, extent, and severity of pavement cracking [7]. Xu and Huang [8] developed an algorithm based on 'grid cell' analysis, which divides the pavement into small cells and a cell is classified as a crack or non-crack based on the statistical characteristics [8]. Ying and Salari [9] proposed a beamlet transform based technique in order to extract linear features such as cracks in pavement after application of an image enhancement algorithm [9]. Recently, there has been a significant improvement in crack recognition approaches that use computer vision techniques.
$\mathrm{Wu}$ et al. [10] developed a crack recognition and segmentation algorithm; the algorithm consists of two steps; (a) using morphological dilation transform to group crack fragments and (b) using thinning transform to connect the fragments. The authors also proposed a method to estimate the crack width for different pavement cracks to characterize the flexible pavements in terms of crack width; the width is the ratio of total area of the fragmented crack pixels to the total length of the connected crack line [10]. Mokhtari et al. [11] used statistical approaches to analyze various features of cracks including area, length, width, orientation, intensity, texture roughness, and wheel path position using computer-vision techniques [11].

Talab et al. [12] proposed a crack detection approach in an image that could accurately segment cracks from noncracks in concrete structures. Otsu method of image thresholding was employed to effectively binarize the image into crack and noncrack pixels [12]. Hoang et al. [13] utilized the technique suggested by Talab et al. [12] for crack detection in flexible pavements. Otsu method of thresholding was used to convert the gray scale image to a monochrome image [13]. This method has the ability to distinct the crack pixels from the surrounding pixels and to separate the original grayscale image into foreground of crack pixels and background of pavement surface.

Though image processing approaches has gained significant interest in assessing pavement distress conditions, researchers still encounter various challenges in image processing due to texture inhomogeneity of pavement aggregates, random noncrack background noises, spots and stains, oils, road markings and so forth. These challenges demand further advancement in image preprocessing and thresholding techniques to precisely assess flexible pavement conditions.

\section{Methodology}

\subsection{Pavement sections selection}

The objective of the study was to identify the features of top-down cracking in flexible pavements. Accordingly, pavement sections with longitudinal cracks in the wheel path were selected for digital image analysis. The design of the pavement and the time of rehabilitation/construction were the main factors in selection of the pavement sections. Table 1 presents the pavement sections used in the analysis.

Table 3. Pavement sections selected for analysis.

\begin{tabular}{|c|c|c|c|c|}
\hline $\begin{array}{c}\text { Control } \\
\text { Section }\end{array}$ & District & Parish & $\begin{array}{c}\text { Construction } \\
\text { Date }\end{array}$ & Route \\
\hline $057-03$ & 3 & 1 & $4 / 24 / 2015$ & LA 13 \\
\hline $058-02$ & 62 & 52 & $12 / 6 / 2012$ & LA 41 \\
\hline $262-03$ & 62 & 32 & $12 / 17 / 2013$ & LA 16 \\
\hline $266-01$ & 61 & 3 & $4 / 26 / 2013$ & LA 22 \\
\hline $829-26$ & 2 & 29 & $7 / 12 / 2013$ & LA 3235 \\
\hline $857-63$ & 3 & 57 & $5 / 28 / 2013$ & LA 82 \\
\hline
\end{tabular}




\subsection{Image analysis}

The pavement images obtained from the LaDOTD Pavement Management System (PMS) were processed to gather significant information related to the longitudinal top-down cracks. The image analysis technique involved three major steps namely image acquisition, image processing, and features extraction as illustrated in Figure 1. Figure 2 presents a sample image of pavement surface used in image processing analysis. In this study, gray-scale images of flexible pavement were analyzed using the ImageJ software. The description of the steps in the image analysis is presented in this section.

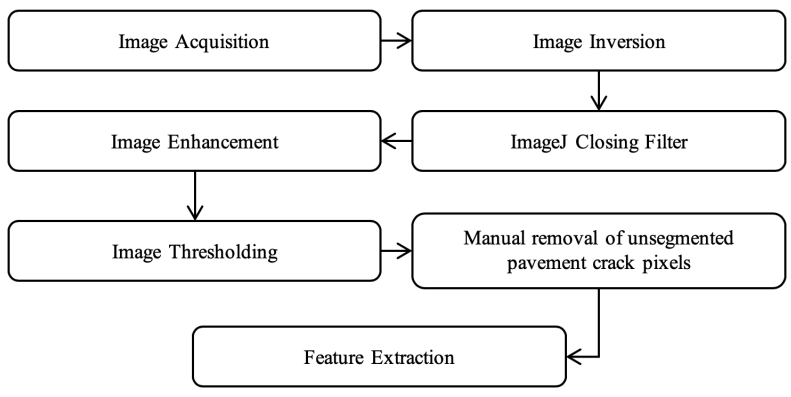

Fig. 1. Image analysis technique.

\subsubsection{Image acquisition}

The images for analysis of the pavement cracks were obtained from the Louisiana PMS inventory. The images are acquired in 8-bit color graphics, which provide 256 distinct levels of gray in which the pixel with a value of 0 is completely black and a pixel with value of 255 is completely white.

\subsubsection{Image processing}

This step involved the application of different enhancement and mathematical operations on an image in order to enhance the visibility of particular features, segment them from the background, and gather the required information. The random backgrounds in pavement images included pavement surface texture, roughness, patches, spots, stains, raveling, and road markings. The pavement images were first inverted, which converted the longitudinal cracks from dark to bright color. The square shaped "Closing" filter was then applied to the inverted image for the noise removal. The "Closing" filter consists of the application of dilation and erosion in succession using the same structuring element for both operations such that the dark structures get smaller and the bright structures (i.e., the pavement cracks) separated by a thin dark space get connected. This filter also preserves the size of the features in the original image. The mathematical morphology defines the closing filter as follows:

$$
A \cdot B=(A \oplus B) \Theta B
$$

where $\mathrm{A}$ is the gray scale image and $\mathrm{B}$ is the structuring element, $\oplus$ and $\Theta$ denote the dilation and erosion, respectively.

The Otsu method (1979) [14] of image thresholding was applied to convert the gray level image to a binary image. This method assumes the images to contain two classes of pixels and iterates through all possible threshold values to compute a measure of spread for the pixels on each side of the threshold such that the original gray scale image is segmented into foreground of crack pixels and background of asphalt pavement [13]. The Otsu method may result in significant error if the sharp valley of the image histogram is degraded by excessive pavement noises. In this study, the image filtering was followed by image enhancement, which involves the adjustment of the filtered image in terms of brightness and contrast so that the Otsu method of thresholding can be efficiently applied. The binarization resulted in segmentation of pavement images into crack and noncrack pixels. It was noticed that the pavement surfaces with the pixels similar to crack pixels still existed after image thresholding. These pavement background pixels were later manually removed to obtain ground-truth image.

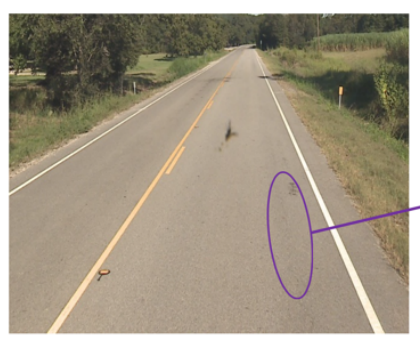

(a)

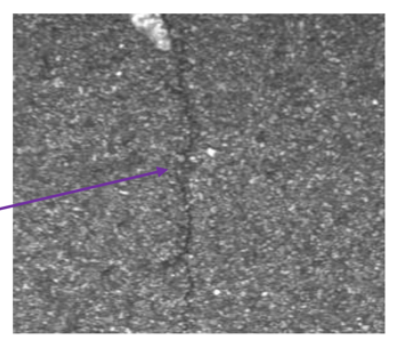

(b)
Fig. 2. Sample of pavement image: (a) lane-scale (b) surfacescale.

\subsubsection{Fracture extraction}

The prominent crack features are extracted based on the binarized images. In this study, three features including crack width, crack intensity, and orientation were extracted and studied.

- Width: The crack width varies along the crack-line path. So, the average crack width is calculated as follows:

$$
\begin{aligned}
& \sum \text { (Width along each } \\
& \text { scan line) } \\
& \sum \text { (Total number of scan } \\
& \text { items) }
\end{aligned}
$$

where the scan lines are automatically defined by the image analysis software based on the pixel size in the image.

- Intensity: This feature measures the intensity of the segmented cracks by overlapping the binarized segmented image into the original image.

- Orientation: The orientation of the cracks was obtained by calculating the angle in degrees between 
the vertical direction and the mid points of the adjacent crack pixels.

\section{Results and discussion}

\subsection{Image processing}

Statistical analysis was performed based on various crack features. The quantitative measures of crack features can be used to estimate the extent and causes of crack deterioration and provide useful information to the state agencies for the required pavement rehabilitation and maintenance strategy. The crack features distribution of pavement cracks for control section 262-03 is presented in Figure 3. This provides a base to segment crack objects from the neighboring pavement surface. The density distribution indicates that the crack intensities are usually in the range of 60 to 160 pixels. The average width of cracks in the pavement after 3 to 5 years of rehabilitation was observed to be 3 to $7 \mathrm{~mm}$. In reference to the vertical longitudinal direction, the cracks were observed to be oriented typically within 20 degrees.

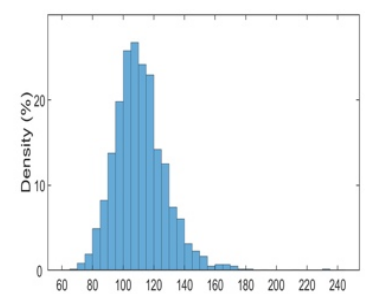

(a)

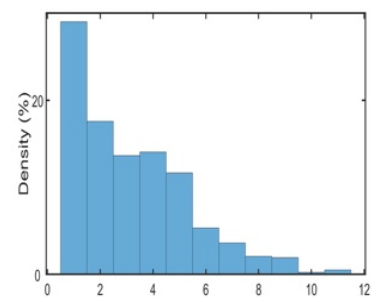

(b)

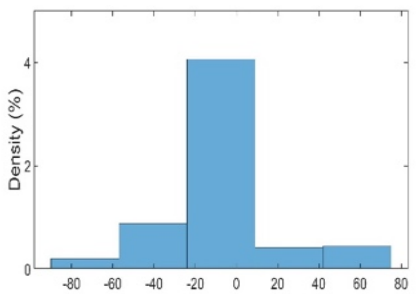

(c)

Fig. 3. Sample statistical distribution of crack features (a) Intensity (b) Width (c) Orientation.

\subsection{Guidelines to identify top-down cracking}

Guidelines were developed to identify top-down cracking based on the characteristics of these cracks and image processing. The top-down crack detection protocol is presented in Figure 4.

\section{Summary and conclusions}

This study presents the evaluation and feature identification of top-down cracking using computervision techniques. The study also reviewed the past literature to characterize the features of top-down cracking in flexible pavements. In order to extract crack features using image processing, six pavement sections images were analyzed using the aforementioned approach. Three different crack features namely width, intensity, and orientation were extracted. The average width and the position of the crack can be considered the optimal features to identify top-down cracking. The intensity and orientation features can have benefits in crack segmentation process in further analysis in the future. Based on the characteristics and image processing results, the following guidelines are recommended to detect top-down cracking in flexible pavements.

- Top-down cracking appear as longitudinal cracking in the wheel path or at the outer edge of wheel path.

- The time frame of appearance of these cracks is within 1 to 10 years of construction. The typical time of appearance is estimated to be 3 to 8 years following construction.

- The crack widths of top down cracking are generally 3 to $7 \mathrm{~mm}$.

- With respect to the orientation of the crack, the cracks segments were mostly longitudinal with typical deviation of 20 degrees.

- The intensity in pavement cracks was observed to be distributed generally within 60 to 160 pixels.

The current study can be expanded to establish further guidelines to identify top-down cracking in flexible pavements. The image processing approach used in the study can be a beneficial tool for crack segmentation and feature extraction, especially in a larger data set that require larger accuracy and computational time. Validation of the proposed methodology is underway using pavement coring.

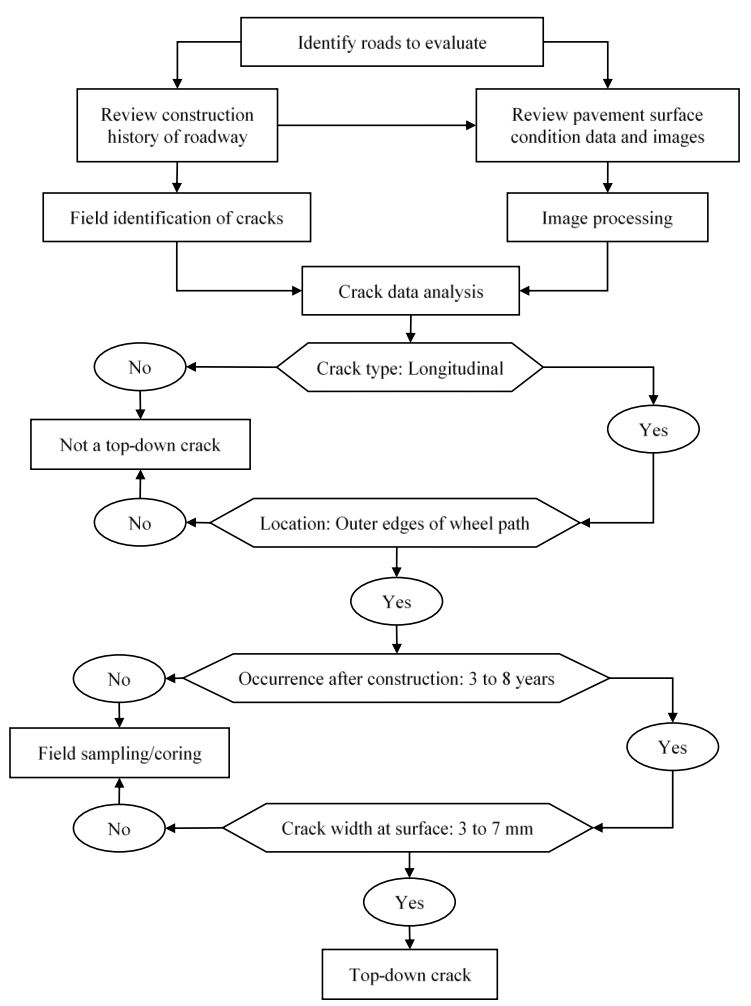

Fig. 4. Top-down cracking identification protocol.

\section{References}


1. Yun, H-B., Mokhtari. S., and Wu, L. (2015). Crack recognition and segmentation using morphological image-processing techniques for flexible pavements. Transportation Research Record: Journal of the Transportation Research Board 2523: 115-124.

2. Chambon, S., and Moliard, J-M., (2011). Automatic road pavement assessment with image processing: review and comparison. International Journal of Geophysics.

3. Svasdisant, T., Schorch, M., and Baladi, G.Y. (2002). Mechanistic analysis of top-down cracks in asphalt pavements. Paper presented in Transportation Research Board Annual Meeting, January, 13-17.

4. Myers, L.A., Roque, R., and Ruth, B.E., (1998). Mechanisms of surface-initiated longitudinal wheel path cracks in high-type bituminous pavements. Journal of the Association of Asphalt Paving Technologists, 67, 401-432.

5. Uhlmeyer, J.S., Willoughby, K., Pierce, L.M., and Mahoney, J.P. (2000). Top-down cracking in Washington state asphalt concrete wearing course. In Transportation Research Record: Journal of Transportation Research Board, No. 1730, TRB, National Research Council, Washington, DC, 110116.

6. Maser, K.R. (1987). Computational techniques for automating visual inspection. Massachusetts Institute of Technology, Report, Cambridge, MA.

7. Georgopoulos, A., Loizos, A., and Flouda, A. (1995). Digital image processing as a tool for pavement distress evaluation. ISPRS Journal of Photogrammetry and Remote Sensing, 50(1), pp. 23-33.

8. Xu, B. and Huang, Y.R. (2003). Development of an automatic pavement surface distress inspection system. No. FHWA/TX-05/7-4975-1.

9. Ying, L., and Salari, E. (2009). Beamlet transform based technique for pavement image processing and classification. In Electro/Information Technology, 2009. eit'09. IEEE International Conference, 141-145.

10. Wu, L., Mokhtari, S., Nazef, A., Nam, B., and Yun, H-B. (2014). Improvement of crack-detection accuracy using a novel crack defragmentation technique in image-based road assessment. Journal of Computing in Civil Engineering, 30(1): 04014118.

11. Mokhtari, S., Wu, L., and Yun, H-B. (2017). Statistical selection and interpretation of imagery features for computer vision-based pavement crack-detection systems. Journal of Performance of Constructed Facilities, 31(5): 04017054.

12. Talab, A.M.A., Huang, Z., Xi, F., and HaiMing, L. (2016). Detection crack in image using Otsu method and multiple filtering in image processing techniques. Optik-International Journal for Light and Electron Optics 127(3): 1030-1033.

13. Hoang, N-D., Nguyen, Q-L., and Bui, D.T., (2018). Image processing-based classification of asphalt pavement cracks using support vector machine optimized by artificial bee colony. Journal of Computing in Civil Engineering, 32(5): 04018037.

14. Otsu, N. (1979). A threshold selection method from gray-level histograms. IEEE transactions on systems, man, and cybernetics, 9(1), 62-66. 